\title{
Typicality effect on N400 ERP in categories despite differences in semantic processing
}

\author{
Mansoureh Fahimi Hnazaee, Marc M. Van Hulle \\ Laboratory for Neuro- and Psychophysiology \\ KU Leuven - University of Leuven \\ Leuven, Belgium
}

\begin{abstract}
We investigate the effect of word typicality -the degree of membership of a word to its superordinate categoryon the N400 event-related potential (ERP) using a single trial detection approach based on spatiotemporal beamforming. Unlike the norm in studies, where mostly concrete categories are used (imaginable objects), we considered a total of 6 basic categories: three abstract and unimaginable (emotion, event, illness), one abstract yet clearly imaginable (color), and two concrete categories, a coherent (mammals) and an incoherent one (furniture). We also investigated the source of the observed N400 ERPs in the brain to detect possible differences between the semantic processing of these categories. Our results show that, independently of word abstractness or concreteness, word typicality has a clear effect on $\mathbf{N 4 0 0}$ both in terms of amplitude and scalp localization as well as in $\mathrm{N400}$ sources, all of which in turn is indicative of differences in difficulty of word processing.
\end{abstract}

Keywords-EEG-ERP, N400 component, prototype theory, word categorization, source localization

\section{INTRODUCTION}

The N400 event related potential (ERP) is commonly used to study language comprehension, mostly word semantics and sentence meaning [1]. The N400, a negative deflection in EEG amplitude, is evoked in response to a stimulus with semantic connotation. In semantic priming, where word pairs are shown in rapid succession, one observes that the N400 in response to the second word is modulated by, among other factors, congruency of the word pair. The N400 effect, as the modulation is called, is believed to result from the activation of word relationships, be it categorical or purely associative. However, some studies argue whether categorical relatedness can be a more reliable predictor than association, or whether relatedness by association produces a larger N400 effect than purely semantic or categorical relatedness [2] [3]. The N400 ERP can be an important ally in unraveling how information, in terms of semantic categories, is processed and stored in the brain, and how this depends on concept modalities such as abstractness and concreteness.

The term categorical relatedness is used to indicate that words are from the same semantic category. A semantic category is usually defined based on two main principles [4]. The first one has to do with the notion that semantic category provides maximum information requiring minimal cognitive effort when confronted with exemplars of that category. The

Mansoureh Fahimi is supported by the Hermes Fund of the National Fund for Scientific Research Flanders (SB/ 151022). MMVH is supported by research grants $\mathrm{PFV} / 10 / 008$, IDO/12/007, IOF/HB/12/021, G088314N, G0A0914N, IUAP P7/11, AKUL 043. second one assures that the perceived word comes as structured information with common and predictable attributes.

Categorization is the ability to determine that a new instance is a member of some known category [5]. Several hierarchical structures for categorization have been proposed, such as the superordinate, basic, and subordinate level of categorization [6]. Among these, the basic level of semantic categorization is of main interest because it is likely to be the level of abstraction that is first learned and recognized by children. EEG studies revealed the differences in N400 effect for different levels of categorization [7].

The ability to categorize is the main pillar in perceiving a relation between a new piece of knowledge and our past experience [8]. A question for ERP research would be to what extent members of the same semantic categories modulate the N400? That is to say: will typical examples (or prototypes) ${ }^{1}$ vs. less common examples (atypical members) lead to differences in N400 ERP? And would this hold despite the fact that different semantic modalities (abstract and concrete) have different spatiotemporal characteristics [32]? Answers to these questions would provide us with an improved insight into how semantic knowledge is categorized in the brain.

According to the prototype hypothesis [6], category membership does not depend on ownership of a set of shared properties, but rather on the evaluation of the resemblance of the candidate to a particular exemplar (prototype) of that category. However, overall members of the same category share a resemblance that is due to the distribution of the most frequent properties of those members [6]. The distance of an exemplar to a prototype, gauged in terms of the number of common features, specifies the typicality of that exemplar" [9] and typical exemplars have shown to evoke a faster response in button press experiments compared to atypical ones [10].

The typicality effect was investigated in a study by Fujihara and co-workers albeit that only concrete categories were used [8]. However, the typicality effect is known to also

\footnotetext{
${ }^{1}$ By typical members or prototypes of a category we mean the clearest cases of category membership, defined operationally by people's judgments of goodness of membership in that category [4]: "an object (abstract or real) that has the greatest number of features in common with other members of a category and the smallest number of features in common with non-member of a category."
} 
hold for ad hoc categories [9]. We know that there is quite a difference in N400 effect for abstract and concrete concepts as they are processed and remembered differently, however, the underlying nature of the difference is still disputed [11]. Concrete words are easier to identify and process [12] contrary to words from abstract categories, the neural substrate of which is still poorly understood [13].

According to embodiment- or sensory-motor theory, the organization of conceptual knowledge is in accordance to the object's semantic features (visual, auditory, action ...) [14]. However, this theory fails to relate to abstract concepts as the latter do not provoke the same sensory experience across subjects. This is an important factor (among others such as defining the degree of abstractness) in explaining why abstract categories are so difficult to investigate, as they are spread out in the brain when activated, as opposed to concrete words that are more localized [13].

The few studies that have been done with N400 report that responses to abstract and concrete words have different temporal and spatial characteristics [13]. In the present paper, unlike earlier studies, we report on a pilot study where we have used word stimuli from both concrete and abstract categories and gauged their effect on the N400. We will also highlight differences in the two categories in terms of source space characteristics. In addition, we differentiate between clearly imaginable (color), coherent (mammals) and incoherent (furniture) concrete words. In our follow-up pilot study, we examined differences of N400 sources between categories. Finally, we examined, for the first time, whether word typicality affects the N400 independently of concept modality (abstract vs. concrete).

\section{MethoDS}

\section{A. Participants}

We recruited for our pilot study 12 subjects, all graduate or under-graduate Flemish-Dutch speaking students (5 males, two left-handed, average age was 21.6 , std=1.9). The data of one subject was discarded due to technical issues during recording, and also that of two others due to too many rejected trials. Furthermore, for our follow-up study, as more subjects are needed to perform source localization, we recruited an additional 13 subjects (total subjects: 9 males, 3 left-handed, average age $=22.5, \mathrm{std}=2$ ). The study was conducted in accordance with the most recent version of the Declaration of Helsinki and approved by the UZ Leuven ethics committee. All subjects signed the written informed consent form prior to participation in the experiment. No participant reported any history on neurological or psychiatric disorders. All subjects were paid for their participation.

\section{B. Materials}

Stimuli were developed to belong to 6 basic categories: 3 groups of clear abstractness (emotion, illness and event), 1 group that is not concrete but still clearly imaginable, and 2 control groups of concrete categories, i.e., one coherent category where membership is very clearly defined and objective (here, mammals) and one less coherent category where membership is subjective (here, furniture). For each of the 6 categories, 15 word stimuli were chosen, resulting in a total of 90 words for in-category word pairs, and a subsequent group of about the same size chosen as fillers from random categories of mainly concrete words (out of category wordpairs). The typical and atypical category members, and also the filler words (non-category members), were matched for word length, orthographic neighborhood size, and frequency of occurrence, using the Dutch CLEARPOND software [15].

Table I. means (m) and standard deviation (std) for word properties

\begin{tabular}{l|l|l} 
& In-category & Non-category \\
\hline Word length & $\mathrm{m}=6.7, \mathrm{std}=2.2$ & $\mathrm{~m}=6.7, \mathrm{std}=1.5$ \\
\hline Orth. Neighb. size & $\mathrm{m}=2.6, \mathrm{std}=3.6$ & $\mathrm{~m}=1.7, \mathrm{std}=2.2$ \\
\hline Freq. of occurrence & $\mathrm{m}=19.2, \mathrm{std}=31.7$ & $\mathrm{~m}=12.1, \mathrm{std}=17.2$
\end{tabular}

We recruited 17 volunteers to score, on a scale of 1-5, 90 words based on how typical they thought an exemplar of each category was. As the scores were typically high, words scoring below 4 were assumed to be atypical and typical otherwise. However, as this threshold resulted in no atypical categories for very inherent categories such as mammals, for those categories, we choose atypical exemplars to be those with minimum score among exemplars.

\begin{tabular}{|c|c|c|c|}
\hline & typical & atypical & nonmember \\
\hline $\begin{array}{l}\text { gebeurtenis } \\
\text { (event) }\end{array}$ & $\begin{array}{l}\text { feest } \\
\text { (party) }\end{array}$ & $\begin{array}{l}\text { kindertijd } \\
\text { (childhood) }\end{array}$ & $\begin{array}{l}\text { basketbal } \\
\text { (basketball) }\end{array}$ \\
\hline $\begin{array}{l}\text { kleur } \\
\text { (color) }\end{array}$ & $\begin{array}{l}\text { blauw } \\
\text { (blue) }\end{array}$ & $\begin{array}{l}\text { amber } \\
\text { (amber) }\end{array}$ & $\begin{array}{l}\text { acteur } \\
\text { (actor) }\end{array}$ \\
\hline $\begin{array}{l}\text { meubel } \\
\text { (furniture) }\end{array}$ & $\begin{array}{l}\begin{array}{l}\text { stoel } \\
\text { (chair) }\end{array} \\
\end{array}$ & $\begin{array}{l}\text { kapstop } \\
\text { (coat rack) }\end{array}$ & $\begin{array}{l}\text { galerij } \\
\text { (gallery) }\end{array}$ \\
\hline $\begin{array}{l}\text { ziekte } \\
\text { (illness) }\end{array}$ & $\begin{array}{l}\text { epilepsie } \\
\text { (epilepsy) }\end{array}$ & $\begin{array}{l}\text { verslaving } \\
\text { (addiction) }\end{array}$ & $\begin{array}{l}\text { ooievaar } \\
\text { (stork) }\end{array}$ \\
\hline $\begin{array}{l}\text { zoogdier } \\
\text { (mammal) }\end{array}$ & $\begin{array}{l}\text { olifant } \\
\text { (elephant) }\end{array}$ & $\begin{array}{l}\text { vleermuis } \\
\text { (bat) }\end{array}$ & $\begin{array}{l}\text { vuilnis } \\
\text { (garbage) }\end{array}$ \\
\hline $\begin{array}{l}\text { emotie } \\
\text { (emotion) }\end{array}$ & $\begin{array}{l}\text { droefheid } \\
\text { (sadness) }\end{array}$ & $\begin{array}{l}\text { verwarring } \\
\text { (confusion) }\end{array}$ & $\begin{array}{l}\text { vliegtuig } \\
\text { (plane) }\end{array}$ \\
\hline
\end{tabular}

\section{Procedure}

The experimental paradigm was a simple word-pair experiment (semantic priming). The prime word was always chosen to be the label of the superordinate category (i.e., the name of the category). The target is randomly chosen to be either a non-member ("filler") or one of the 15 words chosen as member of that category. This procedure was repeated for each category until all 15 stimuli where shown. For each subject, the order of the categories was randomized. Every subject repeated the experiment twice, with a delay of about $20 \mathrm{~min}$. in which they performed another experiment so as to mitigate repetition effects. In total the experiment lasted 30-40 minutes. White words were shown on a black background for 1.2 seconds ( $\pm 200 \mathrm{~ms}$ of jitter). The Psychtoolbox of Matlab was used for stimulus presentation. Prior to each prime-target pair, a fixation cross appeared on the screen requesting subjects to focus in the middle part of the screen. After that, the target stimulus was displayed and a cue was shown 
indicating the subject that he/she had to press the left button of the mouse if they thought the target was a member of the category of which the label shown as prime, or the right button post-onset. The baseline was removed using the average signal in a $100 \mathrm{~ms}$ interval prior to stimulus onset. Trials in which the signal exceeded $\pm 150 \mu \mathrm{V}$ where excluded from the
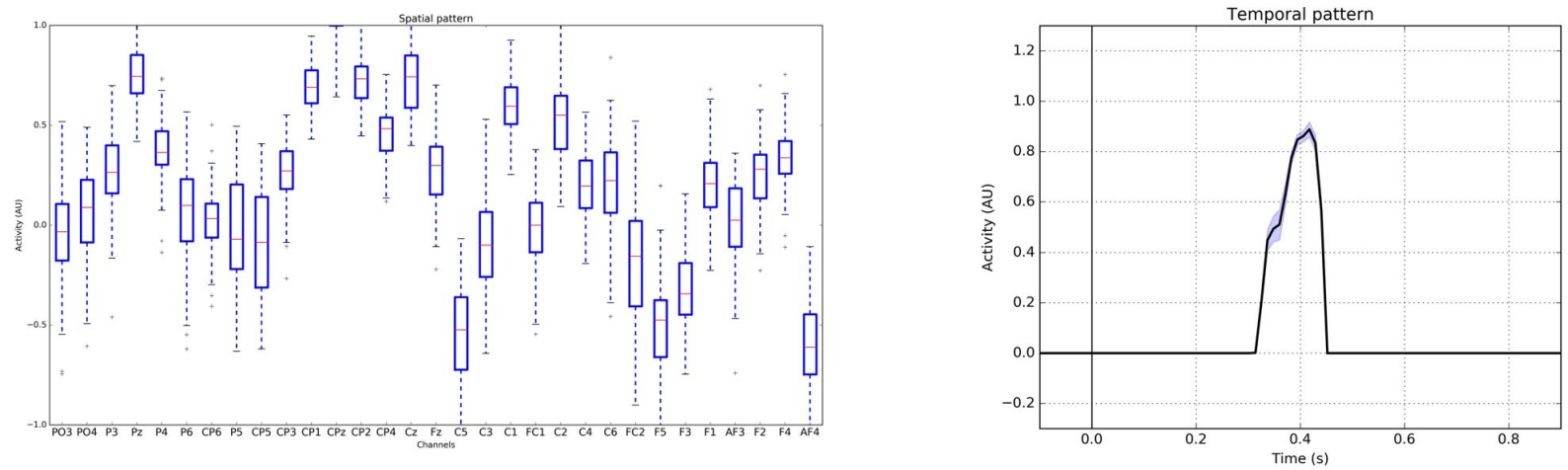

Fig. 1. Boxplot of spatial- (left panel) and temporal template (right panel) of the beamformer in 100 different replications

if otherwise. Subjects were asked not to click the button before the cue appeared so as to prevent contamination of our ERP with motor-related ERPs [16]. The reaction time (RT) of the button press is not relevant to our experiment. After the button press they received visual feedback ("goed!" (correct) for the left button press, and "fout!" (wrong) for the right button press).

\section{Electro-encephalogram (EEG) recording}

Participants were tested in a sound-attenuated, darkened room with a constant temperature of 20 degrees, sitting in front of an LCD screen at a distance of about $70 \mathrm{~cm}$. EEG data was recorded using 128 active $\mathrm{Ag} / \mathrm{AgCl}$ electrodes (SynampsRT, Compumedics, France), according to the international 10-20 system. Two of these electrodes served as ground $(\mathrm{AFz})$ and reference $(\mathrm{FCz})$. The EEG signal was recorded at a $2 \mathrm{KHz}$ sampling rate and downsampled to 250 Hz. All electrodes were mounted in an electrode cap that was placed on the subject's head (Easycap, Germany). This cap is later on used for the predefined channel file and EEG positions in the follow-up study. Conductive gel was applied in each of the electrode holes.

\section{E. Data analysis}

There are several tools available for state of the art preprocessing and analyzing of EEG data [27]. All our data processing (except for the follow-up study) was done with python using prepared packages and the psychic library developed in our lab ${ }^{2}$. The EEG data was re-referenced offline from the original mastoid ground and reference to a common average reference (CAR), and filtered using a $4^{\text {th }}$ order Butterworth filter in the range of $0.1-30 \mathrm{~Hz}$. Analytical tests were performed (checking for variance, kurtosis, and Hurst component) to identify bad channels and to replace them with an interpolation of the surrounding channels. The data was epoched using windows starting from $100 \mathrm{~ms}$ prior to the presentation of the stimulus of interest (target) until $1000 \mathrm{~ms}$

${ }^{2}$ Available online at https://github.com/wmvanvliet/psychic analysis. Trials in which mouse button press responses were incorrect for non-member and typical member targets were omitted, yet incorrect button press responses for atypical words were not omitted. Statistical tests between the errors made in both categories showed no significance, ruling out that any difference in processing found would be due to varying difficulty in decision making between categories. For the follow-up, we preprocessed our data using the aforementioned method, except that we re-rerefenced to the mastoids instead. We performed our source reconstruction analysis using the Brainstorm toolbox [23], which is documented and freely available under the GNU general public license. The default anatomy was selected, and for the forward model we used the OpenMEEG BEM [25]. Bad channels were selected manually for each trial, based on visual inspection of the trials. Noise covariance and data covariance matrices were obtained by merging the matrices calculated from the baseline of all selected trials. For the inverse methods, we used the sLORETA algorithm [26].

\section{F. LCMV Beamformer for single-trial ERP detection}

The linearly constrained minimum variance (LCMV) beamformer [17] is a spatio-temporal filter that relies on spatial- and temporal templates of the ERP collected during a training session (using a proportion of the dataset for training). These templates are formed by subtracting the average of EEG recordings of two experimental paradigms both in time (between 350 and $500 \mathrm{~ms}$ after stimulus onset) and space (electrodes). As our experiment involved three possible outcomes (typical, atypical, and nonmember), we used trials of nonmember and typical targets to maximize the N400 effect. This template is optimized two satisfy two criteria: a) maximal correlation with the actual amplitude of our component of interest (here N400) and b) minimal correlation with interfering signals, such as noise or other ERP components. The template was applied to each epoch separately (single trial) and a single value, regarded as the presence of the N400 in each epoch, retained 


\section{G. Statistical analysis}

Since we have unbalanced data, a linear mixed effect model was used with N400 amplitude (the output resulting of the beamformer, cf. supra) as an independent variable, and with the following fixed effects for several analyses: relatedness (whether or not our target was a member of the category, irrelevant of typicality), typicality (labels of the targets divided into typical, atypical, and nonmembers), and concreteness (labels of the targets divided based on whether they are members of the concrete or the abstract category). Random effects were targets, primes and subjects. Repeated measures analysis of variance (ANOVA) was performed on the outcomes of the linear mixed effect model. For the followup pilot, we performed the data analysis on the source space, in particular on the mean of the source regions of interest, using the independent parametrical tests available in Brainstorm. A significance level of 5\% was adopted for all analyses.

\section{RESULTS}

\section{A. Beamformer results}

Out of the 128 recording channels, we selected a total of 31 channels ('PO3', 'PO4', 'P3', 'Pz', 'P4', 'P6', 'CP6', 'P5', 'CP5', 'CP3', 'CP1', 'CPz', 'CP2', 'CP4', 'Cz', 'Fz', 'C5', 'C3', 'C1', 'FC1', 'C2', 'C4', 'C6', 'FC2', 'F5', 'F3', 'F1', 'AF3', 'F2', 'F4', 'AF4') based on whether their EEG recordings did not cross the $150 \mu \mathrm{V}$ threshold across subjects.

Given that a certain portion of the data should be sacrificed for training our beamformer template, we needed to ensure that it leads to consistent beamformer templates. When we used 60 percent of the data (which is, $30 \%$ of the typical and unrelated trials respectively), we achieved an overall stability in both the spatial and temporal templates which we assessed as follows. (Note that we do not use trials with atypical targets for the formation of the beamformer, because they are expected to be in between the two extreme cases of typical and unrelated, but also because atypical trials in general were less prominent than typical and unrelated ones.) We randomly construed 100 60\% subsets (replications) and determined the mean and variance of the resulting spatial and temporal templates. In fig. 1 we show the boxplot for the spatial template, and the mean and standard error for the temporal template. An example of both temporal and spatial templates is shown in fig. 2 .

The first hypothesis we tested with the beamformer output was on the general relatedness (target versus nontarget). A oneway ANOVA of general relatedness (including typical and atypical members) against unrelated members revealed a significant difference $(\mathrm{p}=0.00175, \mathrm{~F}=5.6743)$. When looking for effects of typicality versus atypicality versus nonmember, a significant difference of $(\mathrm{p}=0.0008435, \mathrm{~F}=4.8172)$ was found, both when all groups were included and when the group 'color' was excluded from the analysis $(p=0.004236, F=3.1828)$. Further pairwise comparison of the groups revealed a significant difference between typical versus atypical exemplars of the categories $(p=0.002725, F=3.7217)$. Inspecting the ANOVA analysis of the effect of concreteness versus abstractness on the N400 amplitude was also significant $(p=0.002589, F=3.0919)$. Note that this result also held when we eliminated the group 'color' from our analysis $(\mathrm{p}=0.0045245, \mathrm{~F}=2.5084)$, showing that our results apply to both cases of using only abstract unimaginable groups, and when the abstract category includes both imaginable and unimaginable words.
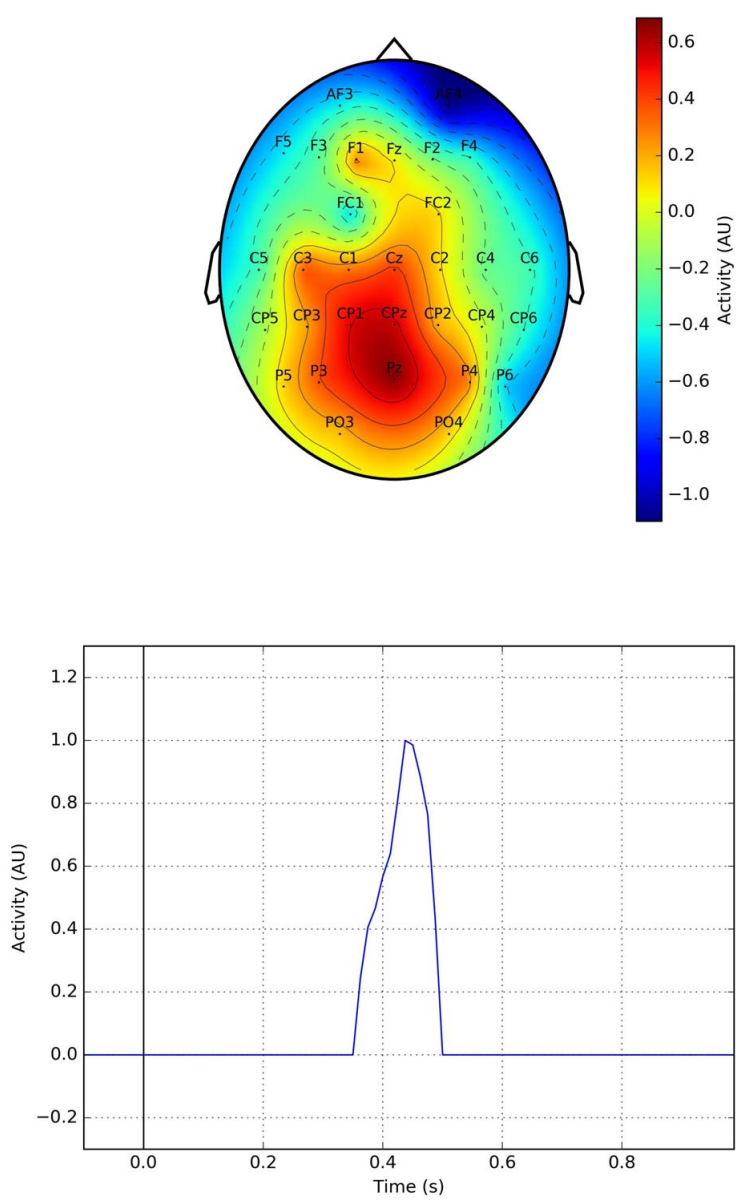

Fig .2. Spatial (up) and temporal (down) beamformer templates

\section{B. ERP analysis}

The ERPs of four centrally located $(\mathrm{Cz}, \mathrm{CPz}, \mathrm{CP} 1, \mathrm{CP} 2)$ electrodes are plotted in fig. 3. They show a clear distinction between the two different groups of categories, contrary to the lateralized electrodes where the abstract and concrete categories resulted in slightly distorted ERPs.

In addition to observing a significant difference in N400 amplitude between the two categories, we now turn to spatial differences. Here we observe a difference (by visual inspection) between abstract and concrete categories. We performed a statistical analysis taking the average values of the channels in each trial over a time period of $350-500 \mathrm{~ms}$ 

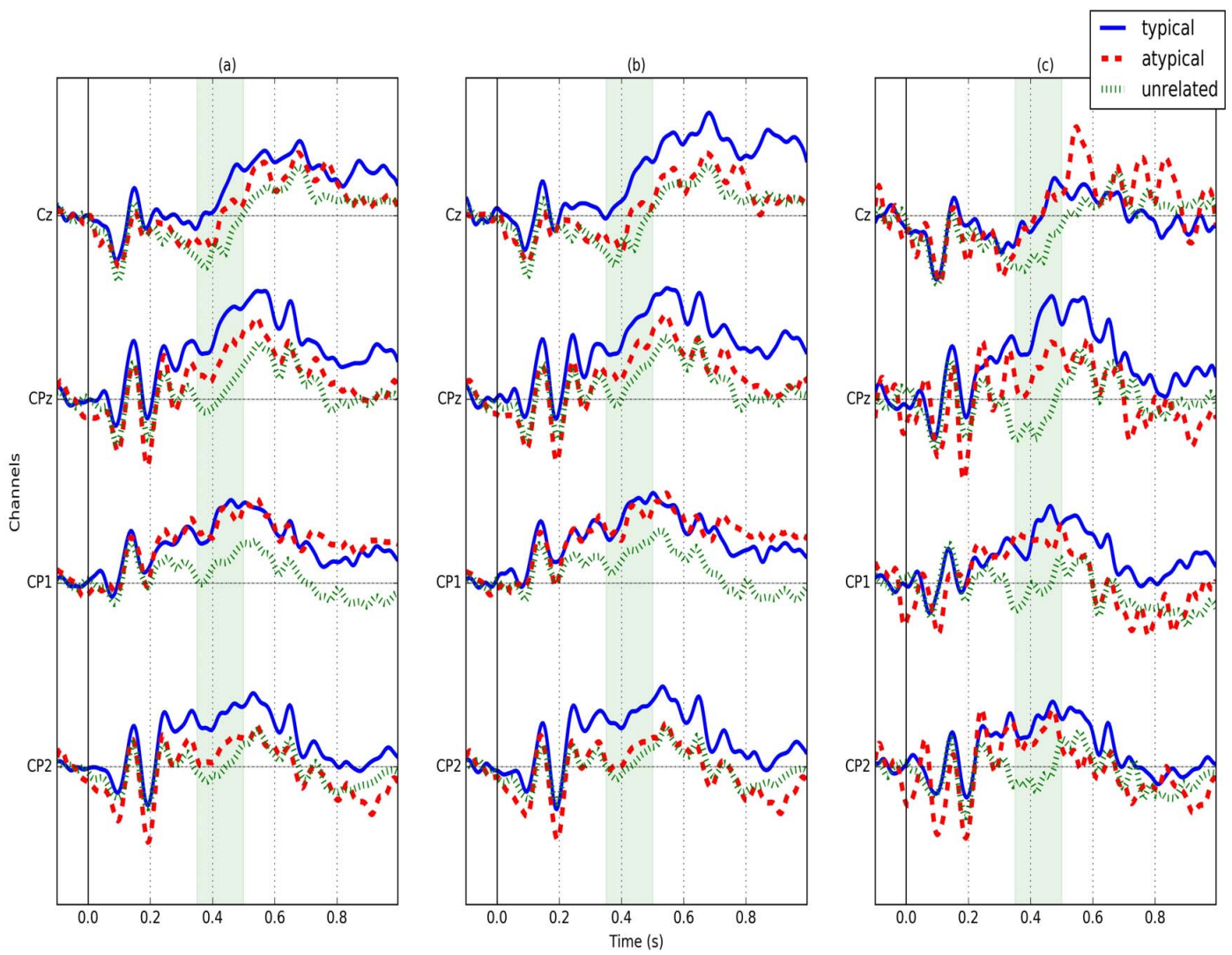

Fig. 3. ERP plots of subjects for a) both categories, b) only abstract categories and c) only concrete categories (EEG activity is plotted such that positive voltages are above $\mathrm{x}$-axis)

(also used for the spatial template) as dependent factor, whereas the modality (abstract or concrete) was used as independent factor, and subject, prime and target were used as random factors. This analysis however revealed no significance in spatial characteristics yet a difference in latency in the ERP plots between the abstract and concrete encouraged can be seen: for example, when looking at the central channel $\mathrm{CPz}$, the latency for concrete categories was smaller (as is expected since they are processed faster). But this finding was not consistent over all channels, therefore, we could not perform a statistical test to confirm the smaller latency for concrete categories.

\section{Source reconstruction analysis}

Source localization is a method to estimate the source of electrical activity measured from the surface of the head, using noninvasive methods such as EEG or MEG. As the N400 is an index of semantic processing, looking into the origins of the source space in this time window can help us understand the underlying neural differences when processing different semantic concepts. The following sections will explain the results seen in the source space between different concepts (a), but also different levels of typicality (b).

\section{a) Semantic categories}

By manually inspecting the differences in sources between the averages of all trials for abstract and concrete trials (from which we excluded the atypical trials in order to show, for the sake of clarity, the difference between extreme cases of both categories), we found several regions of interest. These regions of interest were further used in a statistical test among trials to test for significance. The following regions of interest have shown to be significantly different in the time window of the N400 between the two categories, as can be seen from the figures showing these two categories in fig. 4 and fig. 5.

Among several regions, two regions of interest show significant differences between the two categories. The first one, the posterior part of the middle temporal gyrus on the left hemisphere stands out clearly (shown as the green region in figs. 4 and 5). The posterior middle temporal gyrus (pMTG) is known to be involved in demanding semantic tasks and semantic cognition [24]. Some studies suggest this region as part of a distributed network providing semantic object information with similar patterns for categories such as animals and tools (concrete categories) [28]. Activation of this region was statistically higher for abstract concepts than for concrete ones. This finding is in favor with the dual coding 
model of semantic memory [29], which states that two distinct processing systems are involved in semantic memory: one verbal and one image-based. The verbally mediated system is lateralized to the language dominant (left) hemisphere, and it is this system abstract concepts will heavily depend on for their processing, as abstract words activate image-based processing to a far lesser degree than concrete ones, which would explain the left lateralized network of the abstract concepts.

The second region is the inferior occipital gyrus of the right hemisphere (which can be seen as the red area of fig. 4 and 5). The activation for this region is higher for concrete concepts, which is explained by higher imaginability of concrete concepts exhibited in visual regions, and supported by the literature [30]. These findings support the overall hypothesis of having distinct, yet largely overlapping networks for processing abstract and concrete concepts.

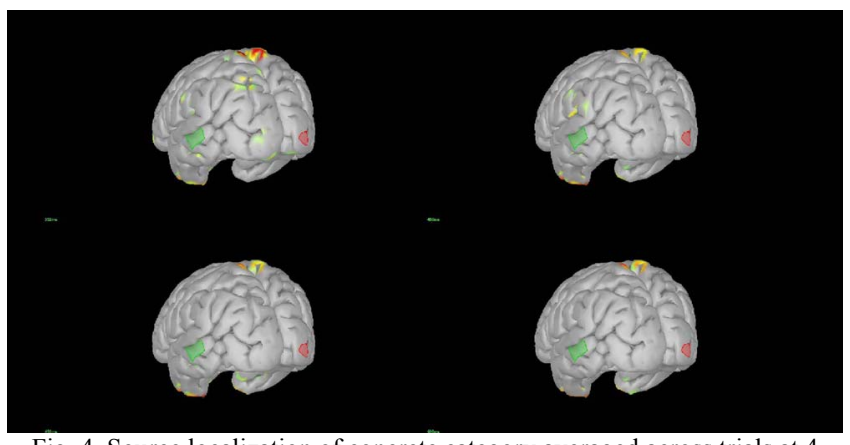

Fig. 4. Source localization of concrete category averaged across trials at 4 time points between $350-500 \mathrm{~ms}$.

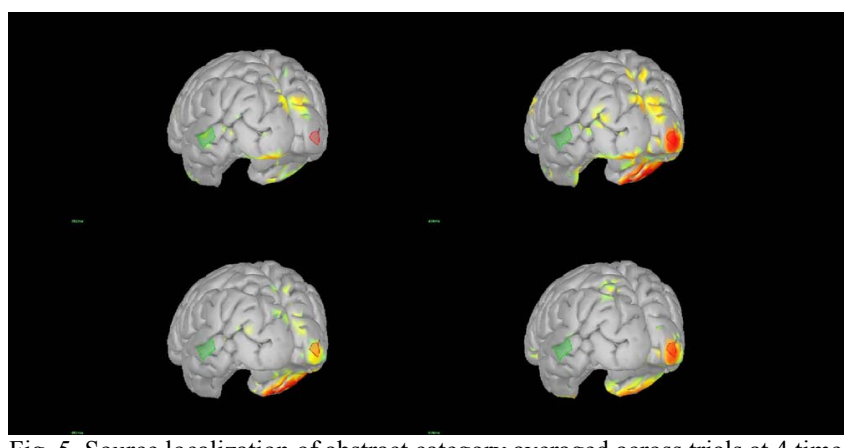

Fig. 5. Source localization of abstract category averaged across trials at 4 time points between $350-500 \mathrm{~ms}$

b) Typicality effects on the source space

Using the same method as explained in the previous section, we found statistical differences in several regions between the typical, atypical and nonmember trials. These regions are shown in figs. 6 to 8 , and summarized in Table III:

Table III. Significant difference in regions of interest between atypical, typical, and unrelated targets

\begin{tabular}{l|l|l} 
& Right hemisphere & Left hemisphere \\
\hline Atypical/typical & -middle region of & - posterior part of \\
& the superior & the superior \\
& frontal gyrus & frontal gyrus \\
& - middle part of & \\
& the middle frontal &
\end{tabular}

\begin{tabular}{l|l|l} 
& $\begin{array}{l}\text { gyrus } \\
\text { - middle part of } \\
\text { the inferior frontal } \\
\text { gyrus } \\
\text { - anterior part of } \\
\text { the } \\
\text { parahippocampal } \\
\text { gyrus }\end{array}$ & \\
\hline $\begin{array}{l}\text { Typical/ } \\
\text { nonmember }\end{array}$ & $\begin{array}{l}\text { - lateral orbital } \\
\text { gyrus }\end{array}$ & $\begin{array}{l}\text { - superior part of } \\
\text { the temporal pole }\end{array}$ \\
\hline $\begin{array}{l}\text { Atypical/ } \\
\text { nonmember }\end{array}$ & $\begin{array}{l}\text { - middle part of } \\
\text { the middle frontal } \\
\text { gyrus }\end{array}$ & $\begin{array}{l}\text { - posterior part of } \\
\text { the intraparietal } \\
\text { sulcus } \\
\text { - superior part of } \\
\text { the temporal pole }\end{array}$ \\
\hline
\end{tabular}

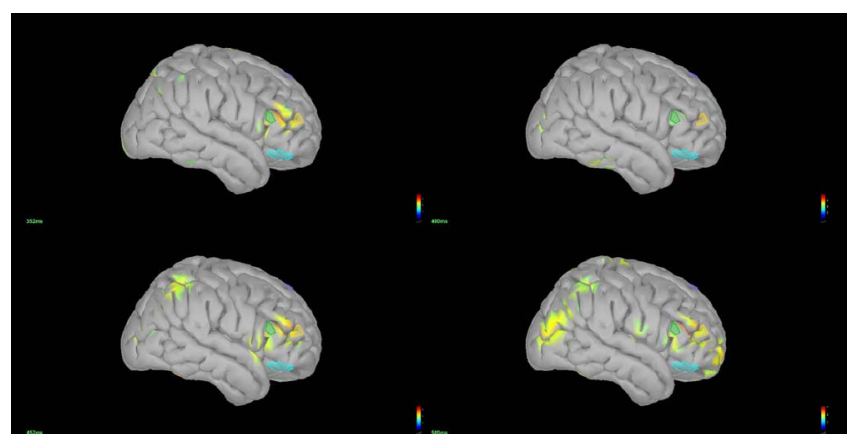

Fig. 6. Source localization of atypical trials averaged across trials at 4 time points between $350-500 \mathrm{~ms}$.

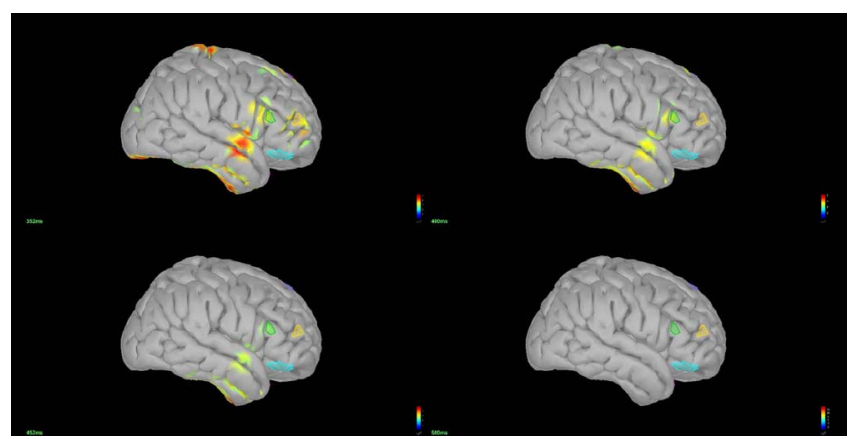

Fig. 7. Source localization of typical trials averaged across trials at 4 time points between $350-500 \mathrm{~ms}$.

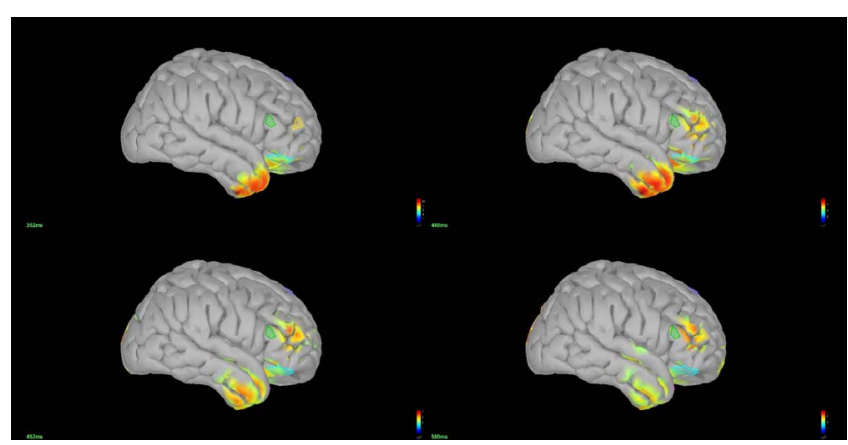

Fig. 8. Source localization of nonmember trials averaged across trials at 4 time points between $350-500 \mathrm{~ms}$ 
It has been hypothesized that the anterior temporal cortex may contribute to successful recognition of orthographical atypical words, whereas typical words are more involving the perisylvian and inferior frontal areas [31]. Also, another study suggests the bilateral temporal cortex to be the most prominent source of the typicality effect, which is supported partially by our results [33]. These suggestions are consistent with our findings, however, further analysis is needed before making any conclusions about the responsible areas for perceiving typicality in a semantic category.

\section{DISCUSSION}

In this study we have investigated the effect of atypical member of a category on the N400 ERP for both abstract and concrete word categories. We observed a bigger N400 effect for trials with less clear examples of a given category, in accordance with the prototype hypothesis [9]. However, this hypothesis was previously tested mostly for categories of imaginable, concrete words, such as vegetables, flowers, or birds [8]. Categories of abstract concepts were given less attention. Here, we evaluated the prototype hypothesis using three abstract categories (illness, event, and emotion) and one abstract- but still imaginable category. The typicality effect was observed in N400 ERP in both the abstract and the concrete categories. The latter has been shown in previous studies [8], but not for the abstract categories. Also it has been shown that the N400 effect is generally larger for concrete versus abstract word-pairs [18] [19]. In this study too we found a significant difference between the two groups, both when the comparison was only between abstract, unimaginable words versus concrete ones, and when the abstract category included imaginable words.

Whether the spatial characteristics of abstract versus concrete words are different or not remains a controversial issue. Neuroimaging studies provide no evidence about the neural underpinnings of concrete versus abstract word processing. [20]. In our ERP study, we failed to see any significant spatial differences between both groups, although our follow-up study confirms differences in source space on the time window of the N400 ERP. However, the N400 amplitude showed a significant difference, and also the N400 latency in response to concrete words seems to be smaller than for abstract words, which is in harmony with the hypothesis that concrete words are processed faster than abstract ones [32].

In previous studies [8], the nonmember category was divided into two groups: related- and unrelated nonmember. However, as shown in [21] the N400 response to category members is not altered by the presence of a nonmember yet related control group. Therefore, as it was not relevant to our main scope, we only used one group as control: the unrelated nonmembers.

\section{CONCLUSION AND OUTLOOK}

A better understanding of the N400 could be used to achieve a more physiologically-motivated classification of words coming from different semantic categories. There is increasing evidence that abstract categories are processed differently in the brain [13]. This is revealed not only in terms of differences in N400 amplitude between categories but also in spatial activation. However, we have shown that the typicality effect for less clear exemplars also holds for several cases of abstract categories. A similar finding has been made by Wang [22], where the typicality of an abstract word was observed to modulate the P2 ERP. Further studies could consider several ERPs jointly to study the typicality effect. Also these studies could take into account models from source reconstruction techniques to get a clearer image of the difference in typicality effect among different modalities of the categories.

We conclude that word typicality affects the N400, independently from concept modality (abstract versus concrete). Our results therefore support the prototype hypothesis [9].

\section{ACKNOWLEDGMENT}

The authors are grateful to (in alphabetic order) Flavio Camarrone, Elvira Khachatryan, Gert Storms and Benjamin Wittevrongel for their helpful comments and suggestions.

\section{REFERENCES}

[1] Kutas, M., \& Federmeier, K. D. Thirty years and counting: Finding meaning in the N400 component of the event related brain potential (ERP).Annual review of psychology, 62, 621, 2011.

[2] Rhodes, S. M., \& Donaldson, D. I. Association and not semantic relationships elicit the N400 effect: Electrophysiological evidence from an explicit language comprehension task. Psychophysiology, 45(1), 50$59,2008$.

[3] Ortu, D., Allan, K., \& Donaldson, D. I. Is the N400 effect a neurophysiological index of associative relationships?. Neuropsychologia,51(9), 1742-1748, 2013.

[4] Rosch, E. Principles of categorization. Etnolingwistyka, (17), 11-35, 2005 .

[5] Markman, A. B., \& Ross, B. H. Category use and category learning.Psychological bulletin, 129(4), 592, 2003.

[6] Rosch, E., \& Mervis, C. B. Family resemblances: Studies in the internal structure of categories. Cognitive psychology, 7(4), 573-605, 1975.

[7] Schumacher, R., Wirth, M., Perrig, W. J., Strik, W., \& Koenig, T. ERP correlates of superordinate category activation. International Journal of Psychophysiology, 72(2), 134-144, 2009.

[8] Fujihara, N., Nageishi, Y., Koyama, S., \& Nakajima, Y. Electrophysiological evidence for the typicality effect of human cognitive categorization. International journal of psychophysiology, 29(1), 65-75, 1998.

[9] Rosch, E. Cognitive representations of semantic categories. Journal of experimental psychology: General, 104(3), 192, 1975.

[10] Kiran, S., Ntourou, K., Eubanks, M., \& Shamapant, S. Typicality of inanimate category exemplars in aphasia: Further evidence for the semantic complexity effect. Brain and Language, 95(1), 178-180, 2005.

[11] Huang, H. W., Lee, C. L., \& Federmeier, K. D. Imagine that! ERPs provide evidence for distinct hemispheric contributions to the processing of concrete and abstract concepts. NeuroImage, 49(1), 1116-1123, 2010

[12] Barsalou, L. W., \& Wiemer-Hastings, K. Situating abstract concepts.Grounding cognition: The role of perception and action in memory, language, and thought, 129-163, 2005.

[13] Kiefer, M., \& Pulvermüller, F. Conceptual representations in mind and brain: theoretical developments, current evidence and future directions. Cortex,48(7), 805-825, 2012.

[14] Martin, A., Ungerleider, L. G., \& Haxby, J. V. Category specificity and the brain: The sensory/motor model of semantic representations of objects.The new cognitive neurosciences, 2, 1023-1036, 2000 
[15] Marian, V., Bartolotti, J., Chabal, S, \& Shook, A. CLEARPOND: Cross-linguistic easy-access resource for phonological and orthographic neighborhood densities. PloS one, 7(8), e43230, 2012.

[16] van Vliet, M., Manyakov, N. V., Storms, G., Fias, W., Wiersema, J. R., \& Van Hulle, M. M. Response-related potentials during semantic priming: the effect of a speeded button response task on ERPs. PloS one, 9(2), e87650, 2014.

[17] Van Vliet, M., Chumerin, N., De Deyne, S., Wiersema, J. R., Fias, W., Storms, G., \& Van Hulle, M. M. Single-Trial ERP Component Analysis Using a Spatiotemporal LCMV Beamformer. Biomedical Engineering, IEEE Transactions on, 63(1), 55-66, 2016.

[18] Kounios, J., \& Holcomb, P. J. Concreteness effects in semantic processing: ERP evidence supporting dual-coding theory. Journal of Experimental Psychology: Learning, Memory, and Cognition, 20(4), 804, 1994.

[19] Tolentino, L. C., \& Tokowicz, N. Are pumpkins better than heaven? An ERP investigation of order effects in the concrete-word advantage. Brain and language, 110(1), 12-22, 2009

[20] Adorni, R., \& Proverbio, A. M. The neural manifestation of the word concreteness effect: An electrical neuroimaging study. Neuropsychologia,50(5), 880-891, 2012

[21] Heinze, H. J., Muente, T. F., \& Kutas, M. Context effects in a category verification task as assessed by event-related brain potential (ERP) measures.Biological psychology, 47(2), 121-135, 1998

[22] Wang, X., Tao, Y., Tempel, T., Xu, Y., Li, S., Tian, Y., \& Li, H. Categorization method affects the typicality effect: ERP evidence from a category-inference task. Frontiers in psychology, 7, 2016.

[23] Tadel, François, et al. "Brainstorm: a user-friendly application for MEG/EEG analysis." Computational intelligence and neuroscience 2011 (2011) : 8.

[24] Noonan, Krist A., et al. "Going beyond inferior prefrontal involvement in semantic control: evidence for the additional contribution of dorsal angular gyrus and posterior middle temporal cortex." Journal of Cognitive Neuroscience 25.11 (2013): 1824-1850.

[25] Gramfort, Alexandre, et al. "OpenMEEG: opensource software for quasistatic bioelectromagnetics." Biomedical engineering online 9.1 (2010): 1.

[26] Pascual-Marqui, Roberto Domingo. "Standardized low-resolution brain electromagnetic tomography (sLORETA): technical details." Methods Find Exp Clin Pharmacol 24.Suppl D (2002): 5-12.

[27] Mahmud, Mufti, and Stefano Vassanelli. "Processing and analysis of multichannel extracellular neuronal signals: state-of-the-art and challenges." Frontiers in Neuroscience 10 (2016).

[28] Chao, Linda L., James V. Haxby, and Alex Martin. "Attribute-based neural substrates in temporal cortex for perceiving and knowing about objects." Nature neuroscience 2.10 (1999): 913-919.

[29] Kounios, John, and Phillip J. Holcomb. "Concreteness effects in semantic processing: ERP evidence supporting dual-coding theory." Journal of Experimental Psychology: Learning, Memory, and Cognition 20.4 (1994): 804.

[30] Sabsevitz, David S., et al. "Modulation of the semantic system by word imageability." Neuroimage 27.1 (2005): 188-200.

[31] Hauk, Olaf, et al. "[Q:] When would you prefer a SOSSAGE to a SAUSAGE?[A:] At about $100 \mathrm{msec}$. ERP correlates of orthographic typicality and lexicality in written word recognition." Journal of Cognitive Neuroscience 18.5 (2006): 818-832.

[32] Binder, Jeffrey R., et al. "Distinct brain systems for processing concrete and abstract concepts." Journal of cognitive neuroscience 17.6 (2005): 905-917.

[33] Hauk, Olaf, et al. "How the camel lost its hump: The impact of object typicality on event-related potential signals in object decision." Journal of Cognitive Neuroscience 19.8 (2007): 1338-1353. 